\title{
The Cape Verde International Days on Mathematics 2017
}

\author{
Delfim F. M. Torres ${ }^{1, *}$, Ricardo Almeida ${ }^{1}$, Paulino Fortes ${ }^{2}$, Dorota Mozyrska ${ }^{3}$ \\ ${ }^{1}$ Center for Research and Development in Mathematics and Applications (CIDMA), \\ Department of Mathematics, University of Aveiro, 3810-193 Aveiro, Portugal \\ 2 University of Cape Verde, Campus de Palmarejo, Praia, Cape Verde \\ ${ }^{3}$ Faculty of Computer Science, Bialystok University of Technology, 15-351 Biatystok, Poland
}

\begin{abstract}
The main contributions of [Stat. Optim. Inf. Comput. Vol. 6, No. 1 (2018)], consisting of nine papers selected and revised from the international conference CVIM'2017, are highlighted.
\end{abstract}

Keywords Optimization; Variational Analysis; Optimal Control; Mathematical Modeling; Fractional Calculus.

DOI: $10.19139 /$ soic.v6i1.464

\section{Introduction}

The Cape Verde International Days on Mathematics 2017 (CVIM'2017), was held at University of Cape Verde, Campus de Palmarejo, from 8 to 11 May, 2017. This conference is part of the research activities of the Systems and Control Group of the Center for Research \& Development in Mathematics and Applications (CIDMA), hosted at the Department of Mathematics of the University of Aveiro, Portugal, and NUMAT - The Research Center in Mathematics and Applications, Faculty of Sciences and Technology, University of Cape Verde, Cape Verde, being the third conference of this series, after CVIM'2013 [1] and CVIM'2015 [2].

CVIM'2017 gathered researchers from several countries to discuss broad topics from Optimization and Variational Analysis, Mathematical Systems Theory, Ordinary and Partial Differential Equations, Geometric Nonlinear Control and Applications, Mathematical Modeling and Numerical Methods Applied to Biomedicine, Matrix theory and its applications, Fractional Calculus and Calculus on Time Scales. There were five plenary lectures and 23 communications on ongoing current research, which play an important role in all fundamental sciences and engineering applications. Simultaneously, there was a "School in Mathematical Modelling and Numerical Methods Applied to Biomedicine", lectured by Maria Neuss-Radu and Adélia Sequeira, for students of the University of Cape Verde.

This SOIC special issue comprises nine original research articles that were carefully selected among works presented at CVIM'2017:

1. A fractional Malthusian growth model with variable order using an optimization approach, by R. Almeida, N. R. O. Bastos and M. T. T. Monteiro;

2. Calculus of variations involving Caputo-Fabrizio fractional differentiation, by N. R. O. Bastos;

3. General quantum variational calculus, by A. M. C. Brito da Cruz and N. Martins;

\footnotetext{
*Correspondence to: delfim@ua.pt (Delfim F. M. Torres).
} 
4. Reduction of forward difference operators in principal $G$-bundles, by A. Casimiro and C. Rodrigo;

5. Variational integrators for reduced field equations, by A. Casimiro and C. Rodrigo;

6. A simple mathematical model for unemployment: a case study in Portugal with optimal control, by A. Galindro and D. F. M. Torres;

7. Quadrature based Broyden-like method for systems of nonlinear equations, by I. A. Osinuga and S. O. Yusuff;

8. Parameter estimation, sensitivity analysis and optimal control of a periodic epidemic model with application to HRSV in Florida, by S. Rosa and D. F. M. Torres;

9. On fractional metric dimension of comb product graphs, by S. W. Saputro, A. Semaničová-Feňovčíková, M. Bača and M. Lascsáková.

Each one of these papers has been carefully scrutinized, having passed the standard refereeing process. Our editorial task was made lighter by the service of unrewarded but dedicated referees: we offer them our heartfelt thanks. We take also this opportunity to thank again all the contributors and participants of CVIM'2017, the authors for submitting their work for possible publication, and the members of the Organizing Committee, for a splendid organization, at all levels, including an enriching social program, which included an excursion to Pico da Antónia, Santiago's tallest mountain located in the central part of the island in Cape Verde; Museum of Tabanka in Assomada, the seat of the municipality of Santa Catarina; and Tarrafal, a town in the northern part of the island of Santiago, known by its beautiful soft-sand and palm-tree lined beach in the shadow of Monte Graciosa. Thanks to the work of all, the meeting was unique and unforgettable. We would like also to acknowledge the generous support of the University of Cape Verde, University of Aveiro, DMat-UA, CIDMA and FCT.

Next, we briefly describe the main contributions of the above mentioned nine papers, in the areas of mathematical modeling and optimization (Section 2), variational analysis (Section 3), and numerical analysis and algorithms (Section 4).

\section{Mathematical Modeling and Optimization}

The paper of Almeida et al. investigates the population growth problem, by modeling its dynamics through a fractional differential equation with the non-integer order being a function depending on time. Using a nonlinear unconstrained optimization problem, the results suggest that, instead of considering constant order fractional differential equations, in some situations it makes sense to consider operators of variable order. Some open questions are pointed out for further investigations.

Rosa and Torres propose a mathematical compartmental model for the Human Respiratory Syncytial Virus (HRSV). Estimation of parameters was done for real data of Florida from September 2011 to July 2014, minimizing the $l_{2}$ norm. The results show that the model fits well the reality under study. Moreover, a sensitivity analysis was carried out, identifying the most important parameters to be taken into account, and an optimal control problem was posed and solved, reinforcing the importance to develop a licensed vaccine for HRSV, which is a subject under strong current investigation.

The article A simple mathematical model for unemployment: a case study in Portugal with optimal control, claims a more realistic and useful model for unemployment, supported with real data from Portugal. An optimal control problem is formulated and solved, providing some non-trivial and interesting conclusions, namely: indirect policies should be the predominant method of avoiding unemployment, whereas the supply of internships should be the main choice when the total level of employment offered is low; governmental policies should be performed mainly during favourable periods, avoiding high unemployment levels in future crisis. 


\section{Variational Analysis}

Bastos studied variational problems that depend on the recent Caputo-Fabrizio derivative operator. Main results provide optimality conditions for several problems of the calculus of variations, which depend on the considered fractional derivative.

In the paper Reduction of Forward Difference Operators in Principal G-bundles, Casimiro and Rodrigo provide the foundations for a larger study of the discretization of variational formulations of field theories that admit symmetries. The focus has been on forward difference operators as the main tool used for the discretization of a Lagrangian. Then, in the companion article Variational integrators for reduced field equations, the authors dedicate themselves to applications in discrete variational principles, presenting variational integrators for Euler-Poincaré equations.

Brito da Cruz and Martins introduce a new variational calculus, based on a general quantum difference operator recently introduced by Hamza et al. They prove necessary optimality conditions of Euler-Lagrange type and natural boundary conditions.

\section{Numerical Analysis and Algorithms}

With the goal to enhance the efficiency of the well known Broyden method, a new quasi-Newton iterative algorithm has been proposed by Osinuga and Yusuff for solving large scale systems of nonlinear equations, reducing the number of iterations it takes to reach a solution. Local convergence analysis and computational results were given.

Saputro et al. consider a graph obtained by the comb product between two connected graphs, proposing a new method for a fractional metric dimension problem.

\section{Acknowledgments}

The authors are grateful to the support of the University of Cape Verde (UniCV), the University of Aveiro (UA), the Department of Mathematics of UA (DMat), and the Center for Research and Development in Mathematics and Applications (CIDMA), project UID/MAT/04106/2013 of the Portuguese Foundation for Science and Technology (FCT). They are also grateful to the local and international Organizing Committees, the Scientific Committee, and all the anonymous referees of the papers submitted for possible publication. Moreover, they deeply thank all the authors for their high quality papers and David Yu and SOIC for all the support to the Special Issue.

\section{REFERENCES}

1. D. F. M. Torres, B. N. Datta, G. S. F. Frederico, N. Martins and A. J. Zaslavski, The Cape Verde International Days on Mathematics 2013, Conference Papers in Mathematics, Hindawi Publishing Corporation, 2013. http://downloads.hindawi.com/journals/proceedings/107850.pdf

2. R. Almeida, G. S. F. Frederico, N. Martins, M. R. Sidi Ammi and D. F. M. Torres, The Cape Verde International Days on Mathematics 2015, Journal of Mathematical Analysis 7(1), 2016. http://www.ilirias.com/ jma/vol_7_issue_1.html 